\title{
Studies on Gene Action and Combining Ability Analysis in Hybrid Rice (Oryza sativa L.)
}

\author{
V. Saikiran ${ }^{2}$, L. Krishna ${ }^{1 *}$, Y. Chandra Mohan ${ }^{1}$ and V. Gouri Shankar ${ }^{2}$ \\ ${ }^{I}$ Department of Plant Breeding, Rice Research Centre, PJTSAU, ARI, Rajendrangar, \\ Hyderabad, Telangana - 500 030, India \\ ${ }^{2}$ Department of Genetics and Pl. Breeding, College of Agriculture, Rajendrangar, Hyderabad, \\ Telangana - 500 030, India \\ *Corresponding author
}

\section{A B S T R A C T}

\section{Keywords}

Gene Action, Combining Ability, Hybrid Rice

(Oryza sativa L.)

Article Info

Accepted:

04 October 2018

Available Online:

10 November 2018
Thirty two hybrids developed from crossing four CMS lines with 8 restorers were studied among with parents for 14 yield and yield attributing characters. Among the male parental lines, JGL 18047, IET 26264 and RNR 26060 appeared the best general combiners for yield and important yield component traits. The most promising specific cross combinations were JMS 13A x RNR 26060 and CMS 64A x WGL 14 for grain yield and most of the component characters.

\section{Introduction}

The success of hybrid rice programme depends on the availability of stable male sterile and restorer lines along with the exploitable level of heterosis and hybrid seed production. In recent years plant breeders have extensively explored and utilized heterosis in boosting up yield of many crops.

However, estimates of heterosis are meagre in rice. The success can further be hastened by choice of suitable parents with favourable alleles which on crossing could produce heterotic hybrids. Rice hybrids developed with the parental lines which are locally adaptable and possessing tolerance to biotic and abiotic stresses are expected to fare well especially in desired environmental conditions. The present investigation aims at assessing the relative importance of general and specific combining abilities effects in breeding programme for some important characters in a $4 \times 8 \mathrm{~L} \times \mathrm{T}$ set of rice.

\section{Materials and Methods}

The experimental material consisted of four WA based cytoplasmic male sterile (CMS) lines and eight elite diverse proven restorer lines, which were crossed in according to the line $\times$ tester mating design. Twelve parents 
along with $32 \mathrm{~F} 1$ hybrids obtained as a result of crosses along with four checks (2 varietal checks MTU 1001 and JGL 11470 and 2 hybrid checks US 312 and HRI 174) grown in at Rice Research Centre, Agricultural Research Institute, PJTSAU, Hyderabad during Kharif 2017.

The experiment was laid out in a Randomized Block Design with two replications. Each treatment consists of two rows of one meter length at a distance of $20 \times 15 \mathrm{~cm}$ spacing. All the necessary recommended package of practices followed to raise the superior hybrids. Five plants of each entry were tagged at random from each plot and data were recorded on the following quantitative characters viz., days to $50 \%$ flowering, plant height, panicle length, number of productive tillers per plant, panicle weight, spikelet fertility (\%), 1000 grain weight, hulling per cent, milling per cent, head rice recovery, kernel length, kernel breadth, kernel lengthbreadth ratio and grain yield per plant. General and specific combining ability effects were calculated following Kempthorne (1957).

\section{Results and Discussion}

The analysis of variance for combining ability (Table 1) revealed that no significant differences were recorded for replications for all the traits. Further, crosses partitioned into lines, testers and lines $\times$ testers. The lines were found significant for certain characters viz., days to 50 per cent flowering, plant height, no. of productive tillers, panicle weight, 1000 grain weight, kernel breadth and lengthbreadth ratio whereas Ramesh et al., (2017) reported significance of the characters viz., plant height, grain length and grain breadth. The testers were significant for 1000 grain weight while the interaction between lines and testers were significant for all the traits except kernel breadth indicating the genetic difference among them.
The estimates of variances due to SCA effects were higher than those due to GCA effects for all the characters except plant height and 1000 grain weight indicating the predominance of non-additive gene action.

The influence of non-additive gene action in the control of yield, yield component traits was also reported by Nadali Bagheri (2010) and Anusha et al., (2017).

CMS 64B and three testers viz., WGL 14, RNR 11450 and IET 26264 recorded high gca values and were found to be good general combiners for grain yield (Table 2). The line CMS 64B also showed high significant gca effects for number of productive tillers per plant, spikelet fertility (\%) and 1000 grain weight. The line CMS 23B and three testers viz., JGL 18047, JGL 11118 and RNR 1140 were good for early flowering and dwarf plant stature.

Among the restorer lines, JGL 18047, IET 26264 and RNR 26060 were identified as best general combiners for yield and important yield components viz., IET 26264 for grain yield per plant, panicle length, number of productive tillers per plant and 1000 grain weight.

Seven crosses viz., JMS 13A x IET 26274, CMS 59A x WGL 14, JMS 13A x RNR 26060, CMS 23A x IET 26264, CMS 64A x WGL 14, CMS 59A x JGL 11118 and CMS 23A x JGL 18047 exhibited ppositive significant sca effects for grain yield (Table 3 ). Among these promising hybrids, CMS 64A $x$ WGL 14 had both the parents with significant positive $\mathrm{gca}$ effects possessing additive $\mathrm{x}$ additive type of gene action. The crosses, CMS 59A x WGL 14 and CMS 23A x IET 26264 possessed only one parent with significant positive $g c a$ effects indicating the involvement of additive and dominance genetic interaction. 
Table.1 Analysis of variance of combining ability for different characters in rice

\begin{tabular}{|c|c|c|c|c|c|c|c|c|}
\hline $\begin{array}{l}\text { Source of } \\
\text { Variation }\end{array}$ & $\begin{array}{l}\text { Degrees } \\
\text { of } \\
\text { freedom }\end{array}$ & $\begin{array}{c}\text { Days to } \\
50 \% \\
\text { flowering }\end{array}$ & $\begin{array}{l}\text { Plant } \\
\text { height } \\
(\mathrm{cm})\end{array}$ & $\begin{array}{l}\text { Panicle } \\
\text { length } \\
\text { (cm) }\end{array}$ & $\begin{array}{l}\text { No. of } \\
\text { Productive } \\
\text { tillers per }\end{array}$ & $\begin{array}{l}\text { Panicle } \\
\text { weight } \\
\text { (g) }\end{array}$ & $\begin{array}{l}\text { Spikelet } \\
\text { fertility } \\
(\%)\end{array}$ & $\begin{array}{c}1000 \\
\text { grain } \\
\text { weight }\end{array}$ \\
\hline Replicates & 1 & 2.91 & 2.32 & 0.45 & 0.27 & 0.01 & 2.42 & 0.03 \\
\hline Treatments & 43 & $93.00 * *$ & $344.27 * *$ & $10.80 * *$ & $7.02 * *$ & $0.29 * *$ & $76.76^{* *}$ & $18.17 * *$ \\
\hline Parents & 11 & 59.01 & 259.38 & 6.75 & 7.31 & 0.15 & 46.41 & 22 \\
\hline Lines & 7 & 90.29 & 368.02 & 8.98 & 6.79 & 0.09 & 52.42 & 25.76 \\
\hline Testers & 3 & 3.46 & 66.83 & 3.03 & 8.95 & 0.23 & 31.75 & 19.97 \\
\hline$L \times T$ & 1 & 6.75 & 76.51 & 2.25 & 6.02 & 0.37 & 48.3 & 1.75 \\
\hline Crosses & 31 & $72.72 * *$ & $382.38 * *$ & $11.11 * *$ & $7.06 * *$ & $0.29 * *$ & $79.68 * *$ & $17.37 * *$ \\
\hline Lines & 7 & $156.14^{*}$ & $998.52 * *$ & 15.35 & $14.61 * *$ & $0.73 * *$ & 92.82 & $37.35 * *$ \\
\hline Testers & 3 & 79.64 & 310.48 & 1.52 & 2.68 & 0.04 & 32.15 & $34.83^{*}$ \\
\hline $\mathrm{L} \times \mathrm{T}$ & 21 & $43.93 * *$ & $187.28 * *$ & $11.07 * *$ & $5.17 * *$ & $0.18 * *$ & $82.09 * *$ & $8.21 * *$ \\
\hline $\begin{array}{l}\text { Parents vs } \\
\text { Crosses } \\
\end{array}$ & 1 & 1095.38 & 96.78 & 45.62 & 2.82 & 1.81 & 320.11 & 0.91 \\
\hline Error & 43 & 1.05 & 7.68 & 1.04 & 1.76 & 0.03 & 1.09 & 0.87 \\
\hline Total & 87 & 46.52 & 173.98 & 5.85 & 4.34 & 0.16 & 38.51 & 9.41 \\
\hline $\begin{array}{l}\text { Source of } \\
\text { Variation }\end{array}$ & $\begin{array}{l}\text { Degrees } \\
\text { of } \\
\text { freedom }\end{array}$ & $\begin{array}{l}\text { Hulling } \\
\text { Per cent }\end{array}$ & $\begin{array}{l}\text { Milling } \\
\text { Per cent }\end{array}$ & $\begin{array}{c}\text { Head } \\
\text { Rice } \\
\text { Recovery } \\
(\%)\end{array}$ & $\begin{array}{c}\text { Kernel } \\
\text { length } \\
(\mathrm{mm})\end{array}$ & $\begin{array}{c}\text { Kernel } \\
\text { breadth } \\
(\mathrm{mm})\end{array}$ & $\begin{array}{c}\text { Kernel } \\
\text { length- } \\
\text { breadth } \\
\text { ratio }\end{array}$ & $\begin{array}{l}\text { Grain } \\
\text { yield per } \\
\text { plant (g) }\end{array}$ \\
\hline Replicates & 1 & 2.53 & 0.33 & 2.54 & 0 & 0 & 0.01 & 4.18 \\
\hline Treatments & 43 & $10.77 * *$ & $12.30 * *$ & $15.68 * *$ & $0.53 * *$ & $0.03 * *$ & $0.19 * *$ & $169.33 * *$ \\
\hline Parents & 11 & 3.87 & 11.73 & 7.49 & 0.59 & 0.04 & 0.28 & 44.42 \\
\hline Lines & 7 & 1.6 & 2.1 & 2.19 & 0.66 & 0.05 & 0.33 & 53.24 \\
\hline Testers & 3 & 10.14 & 32.64 & 5.61 & 0.61 & 0.01 & 0.11 & 21.22 \\
\hline$L \times T$ & 1 & 0.9 & 16.4 & 50.25 & 0.04 & 0.15 & 0.41 & 52.25 \\
\hline Crosses & 31 & $13.48 * *$ & $12.85 * *$ & $16.98 * *$ & $0.48 * *$ & $0.03 * *$ & $0.13^{* *}$ & $178.03 * *$ \\
\hline Lines & 7 & 22.89 & 22.14 & 15.3 & 0.6 & $0.08 * *$ & $0.24^{*}$ & 279.89 \\
\hline Testers & 3 & 6.26 & 6.67 & 2.89 & 0.73 & 0.02 & 0.07 & 95.83 \\
\hline $\mathbf{L} \times \mathrm{T}$ & 21 & $11.38 * *$ & $10.63 * *$ & $19.56 * *$ & $0.41 * *$ & 0.01 & $0.10 *$ & $155.82 * *$ \\
\hline $\begin{array}{l}\text { Parents vs } \\
\text { Crosses } \\
\end{array}$ & 1 & 2.77 & 1.79 & 65.46 & 1.41 & 0.12 & 1.13 & 1273.67 \\
\hline Error & 43 & 1.87 & 4.33 & 4.88 & 0.07 & 0.01 & 0.04 & 10.94 \\
\hline Total & 87 & 6.28 & 8.23 & 10.19 & 0.3 & 0.02 & 0.11 & 89.15 \\
\hline
\end{tabular}

**Significant at $1 \%$ level of significance; * Significant at $5 \%$ level of significance 
Table.2 Estimates of general combining ability effects in lines and testers for yield and yield contributing characters in rice

\begin{tabular}{|c|c|c|c|c|c|c|c|}
\hline Parents & $\begin{array}{c}\text { Days to } \\
50 \% \\
\text { flowering }\end{array}$ & $\begin{array}{l}\text { Plant } \\
\text { height } \\
\text { (cm) }\end{array}$ & $\begin{array}{l}\text { Panicle } \\
\text { length } \\
\text { (cm) }\end{array}$ & $\begin{array}{c}\text { No. of } \\
\text { Productive } \\
\text { tillers per } \\
\text { plant }\end{array}$ & $\begin{array}{l}\text { Panicle } \\
\text { weight } \\
\text { (g) }\end{array}$ & $\begin{array}{l}\text { Spikelet } \\
\text { fertility } \\
(\%)\end{array}$ & $\begin{array}{l}1000 \\
\text { grain } \\
\text { weight } \\
(\mathrm{g})\end{array}$ \\
\hline \multicolumn{8}{|c|}{ LINES } \\
\hline RNR 26060 & 0.172 & $8.295 * *$ & $1.825 * *$ & $-1.756 * *$ & $-0.271 * *$ & $7.151 * *$ & $1.927 * *$ \\
\hline WGL 14 & $5.422 * *$ & $-8.442 * *$ & $-1.238 * *$ & $1.494 * *$ & 0.124 & $2.266 * *$ & -0.165 \\
\hline JGL 18047 & $-6.328 * *$ & $-12.45 * *$ & -0.363 & $-1.069 *$ & $-0.249 * *$ & $-2.138 * *$ & $1.570 * *$ \\
\hline JGL 11118 & $-5.203 * *$ & $-3.442 * *$ & $0.763 *$ & -0.881 & $-0.258 * *$ & $-1.579 * *$ & $-2.443 * *$ \\
\hline RDR 1140 & $-2.578 * *$ & $-4.392 * *$ & $-1.488 * *$ & -0.319 & $0.623 * *$ & $-3.121 * *$ & $0.677 *$ \\
\hline RNR 11450 & $3.547 * *$ & $13.308 * *$ & 0.6 & $2.056 * *$ & 0.112 & $-2.845 * *$ & $-2.192 * *$ \\
\hline IET 26264 & $4.547 * *$ & $16.695 * *$ & $1.575 * *$ & $0.981 *$ & 0.044 & 0.061 & $3.056 * *$ \\
\hline IET 26274 & 0.422 & $-9.567 * *$ & $-1.675 * *$ & -0.506 & -0.126 & 0.204 & $-2.430 * *$ \\
\hline S.E & 0.36 & 0.97 & 0.36 & 0.46 & 0.06 & 0.36 & 0.33 \\
\hline \multicolumn{8}{|c|}{ TESTERS } \\
\hline CMS 23B & $-3.328 * *$ & $-3.136 * *$ & 0.181 & 0.131 & -0.037 & $-1.816 * *$ & 0.458 \\
\hline CMS 59B & $0.859 * *$ & $5.308 * *$ & 0.256 & -0.569 & -0.039 & 0.455 & $0.989 * *$ \\
\hline CMS 64B & $1.047 * *$ & $1.902 * *$ & -0.006 & 0.4 & 0.015 & $1.574 * *$ & $0.742 * *$ \\
\hline JMS 13B & $1.422 * *$ & $-4.073 * *$ & -0.431 & 0.037 & 0.061 & -0.214 & $-2.189 * *$ \\
\hline S.E & 0.25 & 0.69 & 0.25 & 0.33 & 0.04 & 0.26 & 0.23 \\
\hline Parents & $\begin{array}{l}\text { Hulling } \\
\text { Per cent }\end{array}$ & $\begin{array}{l}\text { Milling } \\
\text { Per cent }\end{array}$ & $\begin{array}{c}\text { Head } \\
\text { Rice } \\
\text { Recovery } \\
(\%)\end{array}$ & $\begin{array}{l}\text { Kernel length } \\
\quad(\mathrm{mm})\end{array}$ & $\begin{array}{c}\text { Kernel } \\
\text { breadth } \\
(\mathbf{m m})\end{array}$ & $\begin{array}{c}\text { Kernel } \\
\text { length- } \\
\text { breadth } \\
\text { ratio }\end{array}$ & $\begin{array}{l}\text { Grain } \\
\text { yield per } \\
\text { plant }(\mathrm{g})\end{array}$ \\
\hline \multicolumn{8}{|c|}{ LINES } \\
\hline RNR 26060 & -0.736 & -0.734 & 0.779 & $0.479 * *$ & 0.066 & 0.124 & -10.111 \\
\hline WGL 14 & $-3.619 * *$ & $-2.804 * *$ & $-1.646 *$ & $0.314 * *$ & -0.001 & $0.155 *$ & $4.239 * *$ \\
\hline JGL 18047 & -0.428 & -1.029 & $-2.008 *$ & 0.006 & -0.001 & 0.003 & -0.648 \\
\hline JGL 11118 & $1.665 * *$ & 1.239 & 0.747 & -0.149 & -0.013 & -0.057 & 0.107 \\
\hline RDR 1140 & 0.252 & -0.599 & -0.977 & $-0.216 *$ & -0.056 & -0.017 & -2.106 \\
\hline RNR 11450 & $1.504 * *$ & $2.609 * *$ & 1.11 & $-0.338 * *$ & $-0.115 * *$ & 0.015 & $4.902 * *$ \\
\hline IET 26264 & 0.38 & 0.252 & 0.192 & -0.118 & $0.210 * *$ & $-0.381 * *$ & $8.489 * *$ \\
\hline IET 26274 & 0.981 & 1.067 & $1.804 *$ & 0.022 & $-0.090 *$ & $0.157 *$ & $-4.873 * *$ \\
\hline S.E & 0.48 & 0.73 & 0.78 & 0.09 & 0.03 & 0.06 & 1.16 \\
\hline \multicolumn{8}{|c|}{ TESTERS } \\
\hline CMS 23B & -0.368 & -0.674 & -0.473 & 0.1 & 0.036 & -0.005 & -1.23 \\
\hline CMS 59B & 0.491 & 0.157 & 0.49 & $0.182 * *$ & 0.026 & 0.044 & 1.492 \\
\hline CMS 64B & -0.69 & -0.306 & 0.188 & 0.022 & -0.023 & 0.052 & $2.533 * *$ \\
\hline JMS 13B & 0.568 & 0.823 & -0.205 & $-0.304 * *$ & -0.039 & -0.091 & $-2.795 * *$ \\
\hline S.E & 0.34 & 0.52 & 0.55 & 0.06 & 0.02 & 0.04 & 0.82 \\
\hline
\end{tabular}

** Significant at $1 \%$ level of significance; * Significant at $5 \%$ level of significance 
Table.3 Estimates of specific combining ability effects in hybrids for yield and Yield related traits in rice

\begin{tabular}{|c|c|c|c|c|c|c|c|}
\hline Hybrid & $\begin{array}{c}\text { Days to } \\
50 \% \\
\text { flowering }\end{array}$ & $\begin{array}{c}\text { Plant } \\
\text { height } \\
(\mathrm{cm})\end{array}$ & $\begin{array}{c}\text { Panicle } \\
\text { length } \\
\text { (cm) }\end{array}$ & $\begin{array}{c}\text { No. of } \\
\text { Productive } \\
\text { tillers per } \\
\text { plant }\end{array}$ & $\begin{array}{c}\text { Panicle } \\
\text { weight }(g)\end{array}$ & $\begin{array}{c}\text { Spikelet } \\
\text { fertility } \\
(\%)\end{array}$ & $\begin{array}{c}1000 \\
\text { grain } \\
\text { weight } \\
(\mathrm{g})\end{array}$ \\
\hline CMS 23A x RNR 26060 & 0.703 & -0.539 & $-3.244 * *$ & 0.119 & $-0.572 * *$ & -0.492 & -1.308 \\
\hline CMS 59A x RNR 26060 & -0.484 & -0.233 & 0.081 & -0.431 & 0.096 & $-4.022 * *$ & -0.304 \\
\hline CMS 64A x RNR 26060 & -1.172 & $8.673 * *$ & $2.044 * *$ & -1.9 & 0.046 & -1.202 & 0.803 \\
\hline JMS 13A x RNR 26060 & 0.953 & $-7.902 * *$ & 1.119 & $2.213 *$ & $0.430 * *$ & $5.716 * *$ & 0.809 \\
\hline CMS 23A x WGL 14 & $7.453 * *$ & -1.552 & -0.931 & -1.381 & -0.182 & $2.418 * *$ & -0.536 \\
\hline CMS 59A x WGL 14 & $1.766 *$ & $8.505 * *$ & -0.506 & 0.319 & -0.064 & $6.293 * *$ & $1.503 *$ \\
\hline CMS 64A x WGL 14 & $4.078 * *$ & -0.589 & 0.256 & $2.350 *$ & $0.346 *$ & -1.142 & $1.646 *$ \\
\hline JMS 13A x WGL 14 & $-13.297 * *$ & $-6.364 * *$ & 1.181 & -1.288 & -0.1 & $-7.569 * *$ & $-2.613 * *$ \\
\hline CMS 23A x JGL 18047 & 0.703 & 1.961 & 0.444 & 0.431 & 0.187 & $-3.038 * *$ & -1.181 \\
\hline CMS 59A x JGL 18047 & -0.484 & -1.983 & -0.381 & 0.381 & 0.109 & $7.536 * *$ & -0.127 \\
\hline CMS 64A x JGL 18047 & -0.672 & $-4.327 *$ & $-2.119 * *$ & -1.588 & -0.11 & $-2.628 * *$ & $1.521 *$ \\
\hline JMS 13A x JGL 18047 & 0.453 & $4.348 *$ & $2.056 * *$ & 0.775 & -0.186 & $-1.870 *$ & -0.213 \\
\hline CMS 23A x JGL 11118 & $-2.922 * *$ & $-15.052 * *$ & $-2.931 * *$ & -1.506 & -0.249 & $2.093 * *$ & $2.247 * *$ \\
\hline CMS 59A x JGL 11118 & 1.391 & $8.255 * *$ & $2.744 * *$ & 0.194 & -0.047 & $-6.418 * *$ & -0.959 \\
\hline CMS 64A x JGL 11118 & $-3.297 * *$ & 3.661 & 1.256 & 0.725 & $0.324 *$ & $6.633 * *$ & -0.677 \\
\hline JMS 13A x JGL 11118 & $4.828 * *$ & 3.136 & -1.069 & 0.588 & -0.028 & $-2.309 * *$ & -0.611 \\
\hline CMS 23A x RNR 1140 & $1.953 *$ & $9.348 * *$ & $2.819 * *$ & 0.431 & -0.156 & -0.204 & $3.247 * *$ \\
\hline CMS 59A x RNR 1140 & 1.266 & $-11.795 * *$ & -1.256 & -0.119 & $0.302 *$ & 0.12 & 0.631 \\
\hline CMS 64A x RNR 1140 & $-2.922 * *$ & $-11.139 * *$ & $-1.494 *$ & -0.338 & $-0.433 * *$ & $-6.494 * *$ & $-3.677 * *$ \\
\hline JMS 13A x RNR 1140 & -0.297 & $13.586 * *$ & -0.069 & 0.025 & $0.286 *$ & $6.579 * *$ & -0.201 \\
\hline CMS 23A x RNR 11450 & $-5.172 * *$ & $9.448 * *$ & $2.131 * *$ & 1.306 & $0.501 * *$ & $7.234 * *$ & $-1.535 *$ \\
\hline CMS 59A x RNR 11450 & $2.141 * *$ & $-10.745 * *$ & $-2.244 * *$ & $2.256 *$ & -0.232 & -1.461 & 0.62 \\
\hline CMS 64A x RNR 11450 & -0.047 & 1.161 & $-2.281 * *$ & -0.463 & -0.116 & $8.099 * *$ & $1.952 * *$ \\
\hline JMS 13A x RNR 11450 & $3.078 * *$ & 0.136 & $2.394 * *$ & $-3.100 * *$ & -0.152 & $-13.873 * *$ & -1.037 \\
\hline CMS 23A x IET 26264 & 0.328 & $7.811 * *$ & 1.206 & 0.981 & 0.073 & $-3.417 * *$ & $-2.182 * *$ \\
\hline CMS 59A x IET 26264 & 1.141 & 2.367 & $1.881 *$ & -0.669 & 0.066 & $-1.808 *$ & -1.213 \\
\hline CMS 64A x IET 26264 & -1.047 & $5.773 * *$ & $2.144 * *$ & -1.138 & 0.011 & $-2.927 * *$ & -0.965 \\
\hline JMS 13A x IET 26264 & -0.422 & $-15.952 * *$ & $-5.231 * *$ & 0.825 & -0.15 & $8.151 * *$ & $4.360 * *$ \\
\hline CMS 23A x IET 26274 & $-3.047 * *$ & $-11.427 * *$ & 0.506 & -0.381 & $0.398 * *$ & $-4.594 * *$ & 1.249 \\
\hline CMS 59A x IET 26274 & $-6.734 * *$ & $5.630 * *$ & -0.319 & $-1.931 *$ & -0.229 & -0.24 & -0.152 \\
\hline CMS 64A x IET 26274 & $5.078 * *$ & -3.214 & 0.194 & $2.350 *$ & -0.069 & -0.339 & -0.604 \\
\hline JMS 13A x IET 26274 & $4.703 * *$ & $9.011 * *$ & -0.381 & -0.037 & -0.1 & $5.174 * *$ & -0.493 \\
\hline S.E & 0.72 & 1.95 & 0.72 & 0.93 & 0.12 & 0.73 & 0.66 \\
\hline
\end{tabular}


Contd.,

\begin{tabular}{|c|c|c|c|c|c|c|c|}
\hline Hybrid & $\begin{array}{l}\text { Hulling } \\
\text { Per cent }\end{array}$ & $\begin{array}{l}\text { Milling } \\
\text { Per cent }\end{array}$ & $\begin{array}{c}\text { Head Rice } \\
\text { Recovery } \\
(\%)\end{array}$ & $\begin{array}{c}\text { Kernel } \\
\text { length } \\
(\mathrm{mm})\end{array}$ & $\begin{array}{c}\text { Kernel } \\
\text { breadth } \\
(\mathrm{mm})\end{array}$ & $\begin{array}{c}\text { Kernel } \\
\text { length- } \\
\text { breadth } \\
\text { ratio }\end{array}$ & $\begin{array}{l}\text { Grain } \\
\text { yield per } \\
\text { plant (g) }\end{array}$ \\
\hline CMS 23A x RNR 26060 & -0.079 & -0.732 & -1.514 & 0.186 & -0.088 & 0.236 & $-7.558 * *$ \\
\hline CMS 59A x RNR 26060 & -0.073 & 0.512 & 2.972 & -0.3 & -0.014 & -0.117 & -1.779 \\
\hline CMS 64A x RNR 26060 & 0.538 & 0.874 & 0.274 & 0.15 & 0.041 & -0.001 & -0.771 \\
\hline JMS 13A x RNR 26060 & -0.385 & -0.654 & -1.732 & -0.035 & 0.061 & -0.117 & $10.108 * *$ \\
\hline CMS 23A x WGL 14 & $3.703 * *$ & $3.098 *$ & $5.461 * *$ & -0.039 & 0.064 & -0.135 & -0.307 \\
\hline CMS 59A x WGL 14 & $3.324 * *$ & 2.732 & 2.797 & -0.035 & 0.009 & -0.039 & $14.971 * *$ \\
\hline CMS 64A x WGL 14 & $-6.660 * *$ & $-4.506 * *$ & $-7.801 * *$ & -0.335 & -0.022 & -0.137 & $4.929 *$ \\
\hline JMS 13A x WGL 14 & -0.368 & -1.324 & -0.457 & $0.410 *$ & -0.051 & $0.311 *$ & $-19.593 * *$ \\
\hline CMS 23A x JGL 18047 & $2.427 *$ & 1.203 & 0.873 & 0.078 & -0.041 & 0.107 & $7.280 * *$ \\
\hline CMS 59A x JGL 18047 & -0.132 & -0.093 & -0.54 & -0.343 & -0.066 & -0.061 & -0.642 \\
\hline CMS 64A x JGL 18047 & -0.936 & 0.419 & 2.012 & -0.158 & 0.018 & -0.105 & -3.883 \\
\hline JMS 13A x JGL 18047 & -1.359 & -1.529 & -2.345 & $0.423 *$ & 0.089 & 0.059 & -2.755 \\
\hline CMS 23A x JGL 11118 & -1.926 & -1.356 & -0.622 & 0.238 & 0.016 & 0.087 & -3.405 \\
\hline CMS 59A x JGL 11118 & -0.744 & -1.397 & $-3.385 *$ & $-0.523 * *$ & 0.05 & $-0.341 *$ & $6.353 *$ \\
\hline CMS 64A x JGL 11118 & 0.721 & 0.231 & $3.507 *$ & 0.282 & -0.091 & $0.305 *$ & -1.838 \\
\hline JMS 13A x JGL 11118 & 1.949 & 2.522 & 0.5 & 0.003 & 0.025 & -0.051 & -1.11 \\
\hline CMS 23A $x$ RNR 1140 & -1.138 & -2.212 & $-4.008 *$ & -0.104 & -0.041 & 0.007 & -2.338 \\
\hline CMS 59A $x$ RNR 1140 & -1.162 & -0.123 & -0.522 & 0.21 & -0.026 & 0.154 & $-5.214 *$ \\
\hline CMS 64A $x$ RNR 1140 & $2.439 *$ & 1.474 & 0.505 & $-0.715 * *$ & 0.023 & $-0.400 * *$ & 4.624 \\
\hline JMS 13A x RNR 1140 & -0.139 & 0.861 & $4.024 *$ & $0.610 * *$ & 0.044 & 0.239 & 2.928 \\
\hline CMS 23A x RNR 11450 & 0.896 & $3.399 *$ & 1.425 & -0.303 & -0.032 & -0.1 & 1.53 \\
\hline CMS 59A x RNR 11450 & -1.133 & -2.032 & -1.979 & $0.516 * *$ & 0.073 & 0.141 & 3.158 \\
\hline CMS 64A x RNR 11450 & 0.617 & -0.319 & 1.843 & $0.506 * *$ & 0.042 & 0.203 & 1.267 \\
\hline JMS 13A x RNR 11450 & -0.38 & -1.048 & -1.288 & $-0.719 * *$ & -0.082 & -0.244 & $-5.955 *$ \\
\hline CMS 23A x IET 26264 & -0.301 & 1.257 & 1.173 & $-0.498 *$ & 0.048 & $-0.284 *$ & $10.393 * *$ \\
\hline CMS 59A x IET 26264 & -0.089 & -0.724 & -0.84 & 0.221 & -0.042 & 0.158 & $-4.929 *$ \\
\hline CMS 64A $x$ IET 26264 & 1.091 & -0.262 & -0.538 & $0.381 *$ & 0.007 & 0.149 & $-5.971 *$ \\
\hline JMS 13A x IET 26264 & -0.701 & -0.271 & 0.205 & -0.104 & -0.013 & -0.022 & 0.508 \\
\hline CMS 23A $x$ IET 26274 & $-3.582 * *$ & $-4.658 * *$ & -2.789 & $0.442 *$ & 0.073 & 0.083 & $-5.595 *$ \\
\hline CMS 59A $x$ IET 26274 & 0.009 & 1.126 & 1.497 & 0.256 & 0.017 & 0.105 & $-11.917 * *$ \\
\hline CMS 64A x IET 26274 & $2.190 *$ & 2.088 & 0.199 & -0.109 & -0.018 & -0.014 & 1.642 \\
\hline JMS 13A x IET 26274 & -0.079 & -0.732 & -1.514 & $-0.589 * *$ & -0.073 & -0.175 & $15.870 * *$ \\
\hline S.E & 0.96 & 1.47 & 1.56 & 0.18 & 0.07 & 0.13 & 2.33 \\
\hline
\end{tabular}

Whereas JMS 13A x IET 26274, JMS 13A x RNR 26060, CMS 59A x JGL 11118 and CMS 23A x JGL 18047 crosses had both parents with low gca effects for grain yield per plant indicating over dominance and epistatic interactions. Similar to the present findings, the role of non-additive gene action was documented by other researchers in rice for different traits such as panicle length (Thorat and Kunkerkar, 2017), panicle weight (Ramesh et al., 2017 and Thorat B.S and Kunkerkar, 2017), spikelet fertility percentage (Thorat and Kunkerkar, 2017), grain yield per plant (Dorosti and Monajjem, 2014 and Thorat and Kunkerkar, 2017). 
The CMS line CMS 64B and three testers viz., WGL 14, RNR 11450 and IET 26264 are good general combiners for grain yield. The Hybrids JMS 13A x RNR 26060 and CMS 64A x WGL 14 were best specific combiners for grain yield and yield attributing traits with long slender grain type. The hybrids developed may be further tested extensively in different Agro-climatic zones over seasons for their superiority and stability before commercial release.

\section{References}

Aunsha, Y., Krishna, L., Radhakrishna, K.V and Srinivasa Chary, D. Combining ability analysis for yield and yield component characters in Rice (Oryza sativa L.). 2017. The Journal of Research, PJTSAU 45 (1\&2) 97-100.

Dorosti, H and Monajjem, S. 2014. Gene action and combining ability for grain yield and yield related traits in rice
(Oryza sativa L.). The Journal of Agricultural Sciences. 9 (3): 100-108.

Kempthorne, O. 1957. An introduction to genetic statistics. John Wiley and Sons, Inc: New York.

Nadali Bagheri and Nadali Babaeian Jelodar. 2010 Heterosis and combining ability analysis for yield and related-yield traits in hybrid rice (Oryza Sativa L.). International Journal of Biology. 2 (2): 222-231.

Ramesh, C., Damodar Raju, Ch., Surendar Raju, $\mathrm{Ch}$ and Rama Gopal Varma. 2017. Combining ability and gene action in hybrid rice (Oryza sativa L.). International Journal of Pure and Applied Bioscience. 6 (1): 497-510.

Thorat, B.S and Kunkerkar, R.L. 2017. Study of nature and magnitude of gene action in hybrid rice (Oryza sativa L.) through line $\mathrm{x}$ tester mating design. Contemporary Research in India. 7 (3): 150-154.

\section{How to cite this article:}

Saikiran, V., L. Krishna, Y. Chandra Mohan and Gouri Shankar, V. 2018. Studies on Gene Action and Combining Ability Analysis in Hybrid Rice (Oryza sativa L.). Int.J.Curr.Microbiol.App.Sci. 7(11): 101-107. doi: https://doi.org/10.20546/ijcmas.2018.711.015 